\title{
Processing of natural images is feedforward: A simple behavioral test
}

\author{
Thomas SchmidT AND Filipp SchmidT \\ University of Giessen, Giessen, Germany
}

\begin{abstract}
Natural images can be classified so rapidly that it has been suggested that their analysis is based on a first single pass of processing activity through the visuomotor system. We tested this theory in a visuomotor priming task in which speeded pointing responses were performed toward one of two target images containing a prespecified stimulus (e.g., animal vs. nonanimal, ellipse vs. rectangle). Target pictures were preceded by prime pictures of the same or an opposite category, linked to either the same or an opposite pointing response. We found that pointing trajectories were initially controlled by the primes alone, but independently of information in the actual targets. Our data indicate that prime and target signals remained strictly sequential throughout all processing stages, meeting unprecedentedly stringent behavioral criteria for feedforward processing (rapid-chase criteria). Our findings suggest that visuomotor priming effects capture the output of the very first pass of information through the visuomotor system, before output is affected by recurrent information.
\end{abstract}

Our visual system is able to categorize images of natural visual scenes at a remarkable speed (Hegdé, 2008). Evidence for this has come from studies in which participants were presented with single images and had to indicate as quickly as possible whether or not the image contained an animal, using a go/no-go response (e.g., Thorpe, Fize, \& Marlot, 1996; VanRullen \& Koch, 2003; VanRullen \& Thorpe, 2001, 2002). More recent studies have employed a two-alternative forced choice paradigm in which two pictures were presented side by side and participants had to indicate which one contained the animal (see BaconMacé, Kirchner, Fabre-Thorpe, \& Thorpe, 2007, for a comparison of both types of task). The most spectacular results in this variant of the task have come from a study that required participants to make a rapid eye movement toward the animal picture (Kirchner \& Thorpe, 2006). These authors found that the rate of correct responses began to exceed the rate of errors at saccade latencies as short as 120 msec. Importantly, all of the studies mentioned above were able to trace the transition from nondiscriminative to discriminative processing in the response time distributions of the system's motor output: The fastest responses in distributions are still as likely to be correct as to be incorrect (e.g., Kirchner \& Thorpe, 2006; VanRullen \& Koch, 2003), and the earliest segments of the response time function are unaffected by subsequent visual masking of the target images (Bacon-Macé, Macé, Fabre-Thorpe, \& Thorpe, 2005). These findings suggest that response activation is indeed very fast and direct.

Because of the sheer rapidity of image classification, it has been argued that natural scene processing has to occur during the first pass of information through the visuomotor system (VanRullen \& Thorpe, 2001, 2002). In other words, categorization of natural images, as well as the translation into appropriate motor responses, may be completed within a fast feedforward sweep of visuomotor processing (Bullier, 2001; Lamme \& Roelfsema, 2000). In the context of neuronal signal flow, feedforward indicates that a cell passes activation on to another cell before integrating any feedback or recurrent information from other cells about that signal. During the fast feedforward sweep, a wavefront of visually elicited activation would travel through the visuomotor system so fast that it would essentially be devoid of information from recurrent processing, which would develop only in the wake of the wave (Lamme \& Roelfsema, 2000). Simulation studies of rapid stimulus classification in artificial neuronal networks indeed have suggested that most of the stimulusrelevant information could be extracted from the temporal distribution of the very first spikes in the feedforward wavefront (Serre, Oliva, \& Poggio, 2007; VanRullen, Delorme, \& Thorpe, 2001; VanRullen, Gautrais, Delorme, \& Thorpe, 1998). The issue of feedforward versus recurrent processing is theoretically interesting because many authors have assumed that feedforward processing alone is insufficient to generate visual awareness and that a stimulus must be processed recurrently to become consciously accessible (DiLollo, Enns, \& Rensink, 2000; Fahrenfort, Scholte, \& Lamme, 2007; Lamme, 2002; Lamme, Rodriguez-Rodriguez, \& Spekreijse, 1999; Lamme \& Roelfsema, 2000; Lamme, Zipser, \& Spekreijse, 2002; Pascual-Leone \& Walsh, 2001; Ro, Breitmeyer, Burton, Singhal, \& Lane, 2003; Roelfsema, Tolboom, \& Khayat, 2007; Tong, 2003). 
However, the fact that a single sweep of natural image processing is rapid (or even "ultra-rapid"; VanRullen \& Thorpe, 2001) does not yet imply that it is based on feedforward processing alone. In fact, the hypothesis of a fast feedforward sweep in human vision is controversial, because feedback mechanisms in early visual areas can be very rapid (Bullier, 2001) and there are many possibilities for signals processed in parallel visual streams to cross or overtake each other well before the first overt signs of motor activation (Merigan \& Maunsell, 1993). Still, it would be premature to dismiss the idea of a processing phase that is at least predominantly feedforward; instead, it would be desirable to have a set of behavioral criteria to establish whether the processing system as a whole acts like a feedforward processing system, even if some neuronal feedback might be involved locally.

Because the feedforward sweep is not directly observable, we have proposed a set of behavioral criteria for tasks in which two stimuli presented in rapid sequence (prime and target) try to control the same speeded motor response-for example, a single pointing movement (Schmidt, Niehaus, \& Nagel, 2006; Vath \& Schmidt, 2007). In a simple feedforward system that is reasonably fast and has sufficient temporal resolution, prime and target signals would engage in what we call a rapid chase, with the prime signal maintaining a lead over the target signal all the way to motor control. As a result, the earliest signal arriving in executive motor areas should carry prime-related information exclusively, with no admixture of target information. In other words, visual signals entering the system in strict succession should generate strictly sequential motor outputs. The feedforward properties of the system would show in the time course of the response and meet the following rapid-chase criteria: (1) Prime rather than target signals should determine the onset and initial direction of the response (initiation criterion); (2) target signals should be able to influence the response before it is completed (takeover criterion); and, crucially, (3) response kinematics should initially depend exclusively on prime characteristics and be independent of all target characteristics (independence criterion).

The initiation criterion guarantees that the prime wins the chase; the takeover criterion guarantees that the chase is sufficiently fast to take place within a single response; and the independence criterion establishes strict sequentiality of response control. Stimulus-response systems conforming to these criteria are classified as rapid-chase systems (Schmidt et al., 2006; Vath \& Schmidt, 2007). Note that because of the visuomotor processing delays involved, the sequential response process can unfold after the prime and target stimuli have been presented (and possibly already disappeared). Importantly, note that the rapid-chase criteria do not guarantee that a stimulus-response system is strictly feedforward in all its substages (and they actually do not require such a strict assumption), but they reveal whether a system is behaviorally equivalent to a simple feedforward system. ${ }^{1}$

Rapid-chase processing in speeded choice responses has been demonstrated in a series of studies involving pointing movements to sequential stimuli (Schmidt et al., 2006; Schmidt \& Seydell, 2008; Vath \& Schmidt, 2007) - a variant of the response priming paradigm (Dehaene et al., 1998; Eimer \& Schlaghecken, 1998; Neumann \& Klotz, 1994; Schmidt, 2002; Verleger, Jaśkowski, Aydemir, van der Lubbe, \& Groen, 2004; Vorberg, Mattler, Heinecke, Schmidt, \& Schwarzbach, 2003) — and has also been confirmed in the time course of lateralized readiness potentials (Vath \& Schmidt, 2007). The goal of the present study was to examine the speeded classification of natural images in a paradigm that allowed for evaluation of the rapid-chase criteria, providing a simple and stringent test of the feedforward account advocated in earlier studies.

We compared four tasks in two experiments to demonstrate rapid-chase processing of natural images. In each task, two target images (each from one of two picture categories-e.g., animals and objects) appeared in diagonally opposite quadrants of the display (Figure 1A). Participants pointed from the center of the display toward the picture of the appointed category (e.g., the animal). Just previous to the targets, there were two prime images, one from each category, at the same two positions. The primes appeared for $33 \mathrm{msec}$ at prime-target stimulus onset asynchronies (SOAs) of 33, 50, 67, 83, or $100 \mathrm{msec}$. Either the prime and target image categories could correspond (consistent trials), or the prime image categories could be switched with respect to the target image categories (inconsistent trials). Consistent and inconsistent primes were expected to initiate responses in the direction of the correct target image or in the opposite direction, respectively. We employed the following tasks.

1. In the animal-object task in Experiment 1, image categories were animals and nonanimals presented as grayscale photographs from the large COREL image database used in most earlier studies on rapid image classification. The participants pointed as quickly as possible toward the animal target. This task was designed to match the standard conditions in most studies of rapid image classification (Bacon-Macé et al., 2007; Bacon-Macé et al., 2005; Kirchner \& Thorpe, 2006; Thorpe et al., 1996; VanRullen \& Koch, 2003; VanRullen \& Thorpe, 2001, 2002) and, particularly, to translate the task employed by Kirchner and Thorpe (2006) into a priming task with speeded pointing movements that could be used to evaluate the rapidchase criteria.

2. In the large-small task in Experiment 1, the same pictures were presented, but the participants had to point toward the item that would be larger in real life (regardless of animality). We expected that this classification would be too difficult and too slow to be based on feedforward processing and, thus, would defy the rapid-chase criteria.

3. The toy-animal-object task in Experiment 2 was identical to that in Experiment 1 but used novel stimuli. These were chosen because they appeared in high color and luminance contrast against a black background, potentially allowing for faster classification than the grayscale COREL images that depicted animals and objects at lower luminance contrast in front of their natural cluttered backgrounds. We hoped that the color images would provide a stronger feedforward signal and would yield larger priming effects than those observed in Experiment 1. 

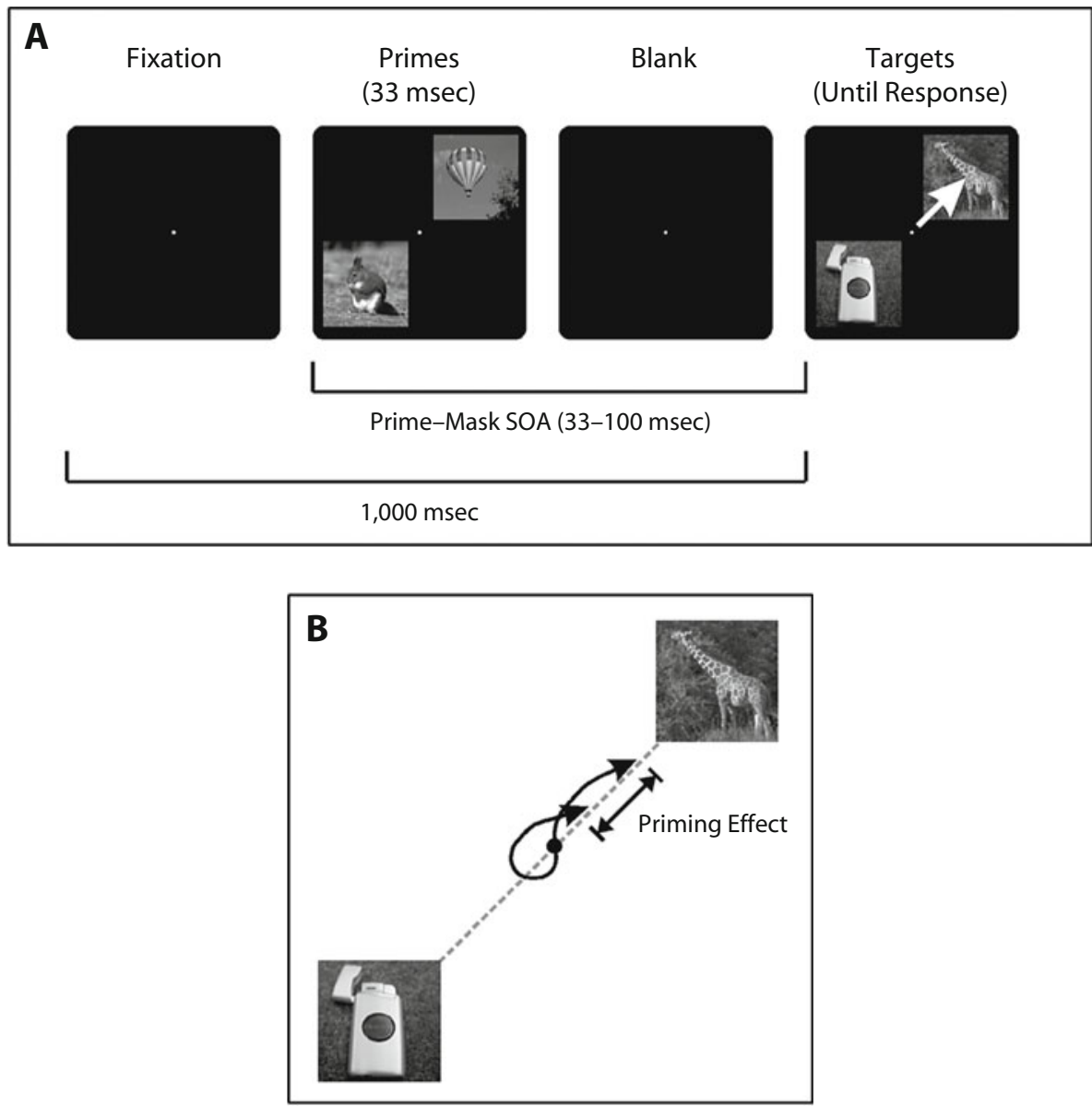

\section{C}

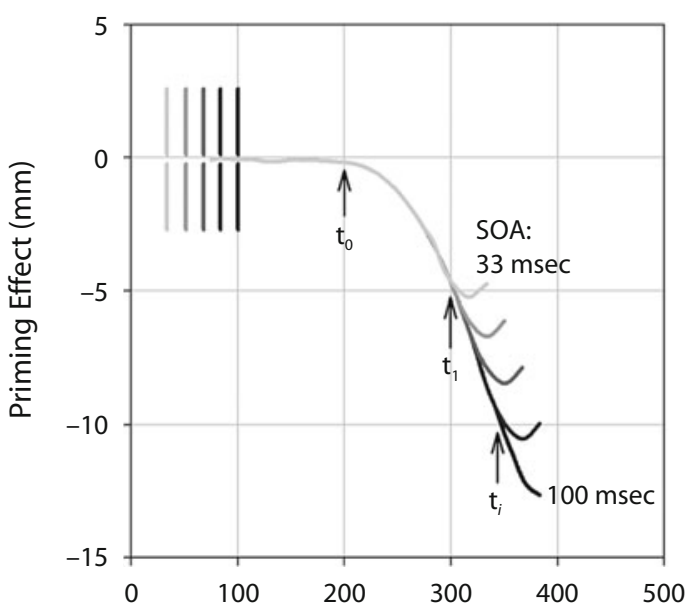

D

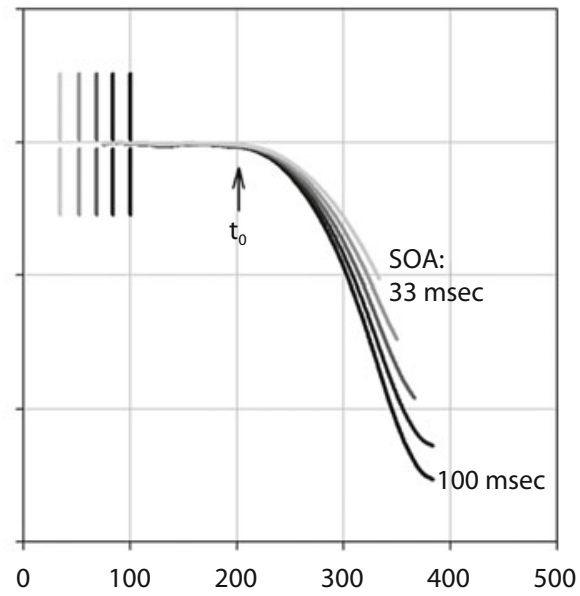

Time From Prime Onset (msec)

Figure 1. Experimental paradigm. (A) Time course of a trial. Note that different stimuli were used in different conditions in Experiments 1 and 2. The arrow illustrates a correct pointing movement. (B) Definition of spatial priming functions. Sensor positions in consistent and inconsistent trials were projected onto the target-nontarget axis and were subtracted. The resulting measure of priming is negative when the sensor position in consistent trials leads the sensor position in inconsistent trials. (C) Rapid-chase theory predicts that spatial priming functions should initially be independent of prime-target SOA, so that all priming functions should follow the same initial time course. (D) Generally, in a non-rapid-chase system, prime and target signals would mix prior to movement onset, leading to a fanning up of priming functions right from the start of the movement. Short vertical lines indicate target onsets at different prime-target SOAs; shading differentiates the respective SOAs. 
4. In the ellipse-rectangle task in Experiment 2, one ellipse and one rectangle were presented as targets (preceded by one ellipse and one rectangle as primes), and the participants had to point toward the ellipse. This task was expected to be fast and easy enough to approximate an upper limit on classification speed against which the animal-object tasks could be compared. Of course, we expected it to yield unequivocal evidence for rapid-chase processing.

To evaluate the rapid-chase criteria, we examined the time course of priming effects defined in the spatial domain - that is, the spatial lag of the pointing movements in inconsistent, as compared with consistent, trials at corresponding points in time (see Figure 1B for a definition). In a rapid-chase system with sufficient temporal resolution, prime and target images would be processed in strict sequence with no temporal overlap. In the earliest phase of detectable motor output, the prime signal alone should drive the response, generating a priming effect completely unchecked by information in the actual target. Only after some time, depending on SOA, would the target signal start to take over the response, diminishing the priming effect. As a consequence, spatial priming effects would have identical early time courses when time-locked to prime onset and would only branch off from the common time course at different points in time, depending on the prime-target SOA. Specifically, for each pair of SOAs $i$ and $j$, where $\mathrm{SOA}_{i}<\mathrm{SOA}_{j}$, the time course of spatial priming effects should be invariant between the priming effect's onset time, $t_{0}$, and the branch-off time for the shorter SOA, $t_{i}$ (Figure $1 \mathrm{C}$ ). In contrast, in a more general system in which prime and target signals would undergo some mixture prior to $t_{0}$, the priming effect should be progressively smaller for larger influences of the target signal relative to the prime signal - that is, for shorter SOAs. As a result, the time course of spatial priming effects should differ right from $t_{0}$, leading to an immediate fanning up of spatial priming functions (Figure 1D). ${ }^{2}$

\section{METHOD}

\section{Participants}

Twelve students from the University of Giessen (Experiment 1, 6 participants, 20-29 years of age, 1 male, 1 left-handed; Experiment 2, 6 participants, 21-30 years of age, 2 male, all right-handed) volunteered for course credit or for a payment of $€ 8 / \mathrm{h}$. Their vision was normal or appropriately corrected. All of them gave informed consent.

\section{Apparatus}

The experiment was controlled by a $300-\mathrm{MHz}$ PC driving a 14in. VGA color monitor $(640 \times 480$ pixels $)$ in synchrony with the monitor retrace rate of $60 \mathrm{~Hz}$. The monitor image was projected onto a workspace via a set of two mirrors, so that the participants had the impression that the stimuli appeared directly on the workspace, where they could interact with them. The workspace was tilted toward the participant by $60^{\circ}$ out of the transversal plane at a $80-\mathrm{cm}$ viewing distance and was illuminated so that the participants could view their hand superimposed on the stimuli. Pointing responses were recorded by a Polhemus FASTRAK magnetic tracking device at a sampling frequency of $120 \mathrm{~Hz}$. The magnet sensor was located in a hand-held stylus, referenced to the tip of the stylus.

\section{Stimuli}

The primes were small images $(45.3 \times 45.3 \mathrm{~mm} ; 100 \times 100$ pixel at $72 \mathrm{dpi}$, in 8-bit color or grayscale) presented against a dark background $\left(0.07 \mathrm{~cd} / \mathrm{m}^{2}\right), 36.2 \mathrm{~mm}$ from a $83.00-\mathrm{cd} / \mathrm{m}^{2}$ fixation point $\left(0.9 \times 0.9 \mathrm{~mm} ; 1 \mathrm{~mm} \approx 0.032^{\circ}\right.$ of visual angle). The targets were images of the same size occurring at the same positions as the primes. The pictures in Experiment 1 (animal-object and large-small tasks) were selected from the COREL image database. We used 40 animal and 40 object images, half of which could be classified as small (up to the size of a duck or a bucket) or large (down to the size of a donkey or a car). In the toy-animal-object task in Experiment 2, the pictures were chosen from the Amsterdam Library of Object Images (Geusebroek, Burghouts, \& Smeulders, 2005). The 20 animal images were pictures of cuddly toys and dolls, whereas the 20 object images mainly depicted household items. For the ellipse-rectangle task in Experiment 2, we constructed 20 ellipses and 20 rectangles in one of two orientations $\left( \pm 45^{\circ}\right)$ and 10 different aspect ratios (1:1.23-1:5.25); they appeared in gray against a black image background.

In the animal-object task in Experiment 1, each of the prime and target pairs contained one animal and one object image, each of which was randomly chosen from the pool of all the animal or object images. Selections were made regardless of largeness, so that two large or two small images could serve as the prime or target pairs. Similarly, in the large-small task, each of the prime and target pairs contained one large and one small animal or object image, with selections being made regardless of animality. In the toy-animalobject and ellipse-rectangle tasks in Experiment 2, each of the prime and target pairs contained one animal and one object (or one ellipse and one rectangle) image, each of which was randomly chosen from a pool of 20 pictures for each category.

\section{Procedure}

The experimental procedure was identical in all four tasks. The participants placed the stylus on the fixation point at display center and pressed the space bar to initiate each trial (Figure 1A). They were then presented with prime images flashed simultaneously for $33 \mathrm{msec}$ in diagonally opposite quadrants of the display. The pair of quadrants was selected randomly to discourage anticipation strategies. After a prime-target SOA of 33, 50,67, 83, or $100 \mathrm{msec}$, two target images were presented at the same locations as the primes. Targets remained on the screen until the participants had finished a speeded pointing response toward either target. The response was considered finished when the sensor entered a sphere of $17-\mathrm{mm}$ radius around any of the four target positions for at least $50 \mathrm{msec}$.

The participants were instructed to point as quickly as possible to the correct target. In consistent trials, primes and targets from the same category were presented at corresponding positions; in inconsistent trials, prime positions were switched. Stimulus conditions occurred equiprobably and pseudorandomly in a completely crossed repeated measures design. In each experiment, the participants took part in eight experimental sessions, four in each of the two respective tasks, with task order counterbalanced across participants. Each session consisted of 1 warm-up block, followed by 15 experimental blocks of 40 trials, and the participants were debriefed after the final session and received an explanation of the experiment.

\section{Data Treatment and Statistical Methods}

From both experiments combined, 22 blocks (out of 1,440) were lost due to equipment malfunction. Warm-up blocks were not analyzed. Trials were excluded if the participant had hit one of the empty, off-diagonal target locations or if arrival times were shorter than $100 \mathrm{msec}$ or longer than $999 \mathrm{msec}$. This procedure eliminated $1.14 \%$ and $1.73 \%$ of the trials in Experiments 1 and 2, respectively.

Pointing trajectories were simplified by projecting the horizontal and vertical coordinates of the stylus tip onto a line connecting the target and the nontarget, defining the starting point as zero and the correct target direction as positive. We looked at priming effects in the temporal as well as the spatial domain. For temporal priming 
A

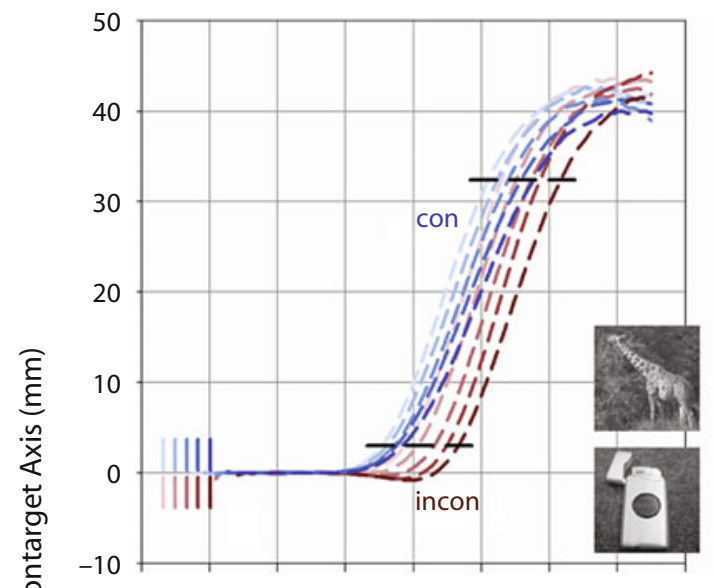

B

Exp. 1: Large vs. Small

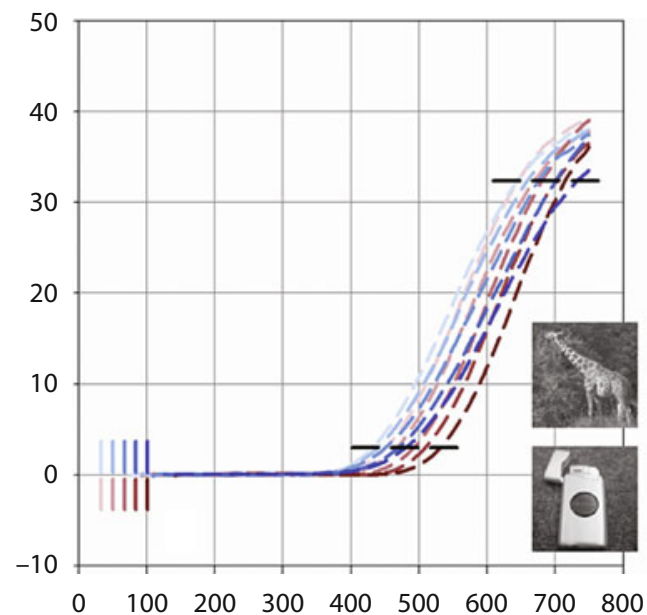

\section{Exp. 2: Toy Animal vs. Object}

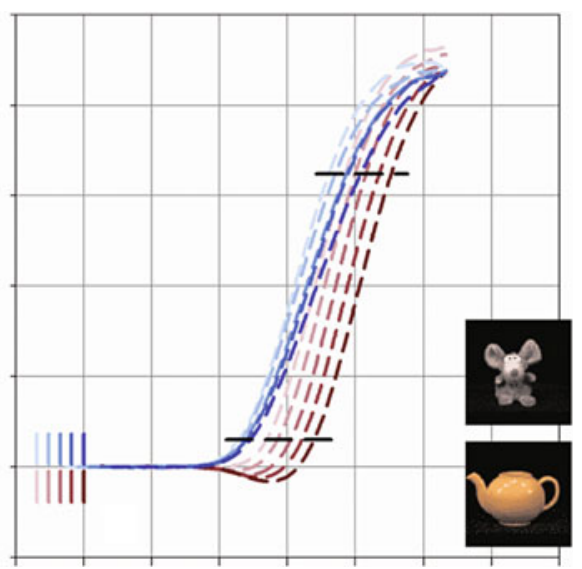

D Exp. 2: Ellipse vs. Rectangle

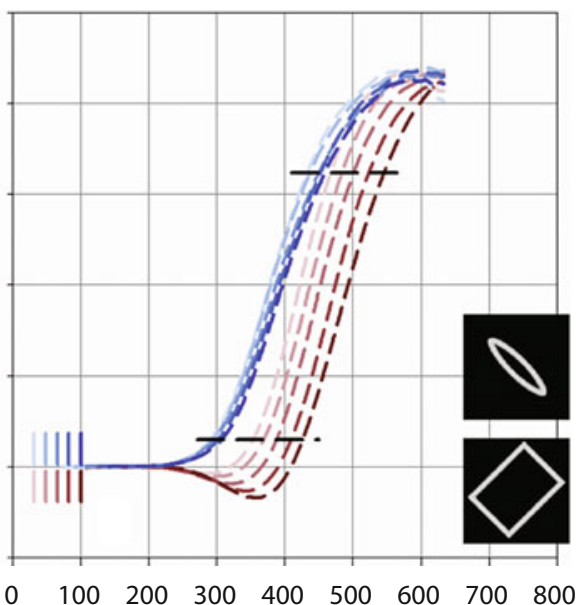

Time from Prime Onset (msec)

Figure 2. Pointing trajectories in the four classification tasks in Experiments 1 and 2. Only correct responses are shown. Positive position values indicate movement toward the correct target; negative values indicate movement in the opposite direction. Note that trajectories are time-locked to prime onset to emphasize that the movement starts at a fixed time following prime onset and initially goes in the direction specified by the primes. Short vertical lines indicate target onset at prime-target SOAs of 33-100 msec; the shading (color code online) serves to differentiate trajectories at the respective SOAs. Horizontal bars mark early and late criteria for evaluating priming effects in the temporal domain. Insets show sample stimuli.

effects, we compared the times when consistent and inconsistent trajectories crossed various spatial criteria. In addition, we defined a spatial priming function by subtracting trajectories in consistent trails from those in inconsistent trials (negative values indicating how far the sensor position in inconsistent trials lagged behind the sensor position in consistent trials at corresponding points in time; see Figure 1B)

Kinematic parameters of trajectories and spatial priming functions were extracted by jackknifing methods (Ulrich \& Miller, 2001). Pointing trajectories of the $n$ participants were averaged across each subsample of $(n-1)$ participants, excluding a different participant from each subsample. Averaged trajectories in the subsamples were then smoothed with a T4253H filter (Velleman, 1980). The smoothing filter had no detectable influence on the averaged trajectories but considerably smoothed their time derivative, which was helpful in extracting velocity parameters. Movement parameters (e.g., arrival times and peak velocities) were estimated from each subsample and then averaged. This elegant procedure allows for estimates that are much more reliable than those from single participants, without altering the average trajectories (Ulrich \& Miller, 2001). Finally, $F$ tests and standard error bars were corrected to estimate variation among participants, rather than among subsamples.

\section{RESULTS}

\section{Experiment 1: Animal-Object and Large-Small Tasks}

Figure 2 shows how the primes affected the trajectories of pointing responses to the correct target. After the primes and targets had appeared, the sensor remained at rest for a while. In consistent trials, it started to move toward the correct target roughly $300 \mathrm{msec}$ after prime onset in the 


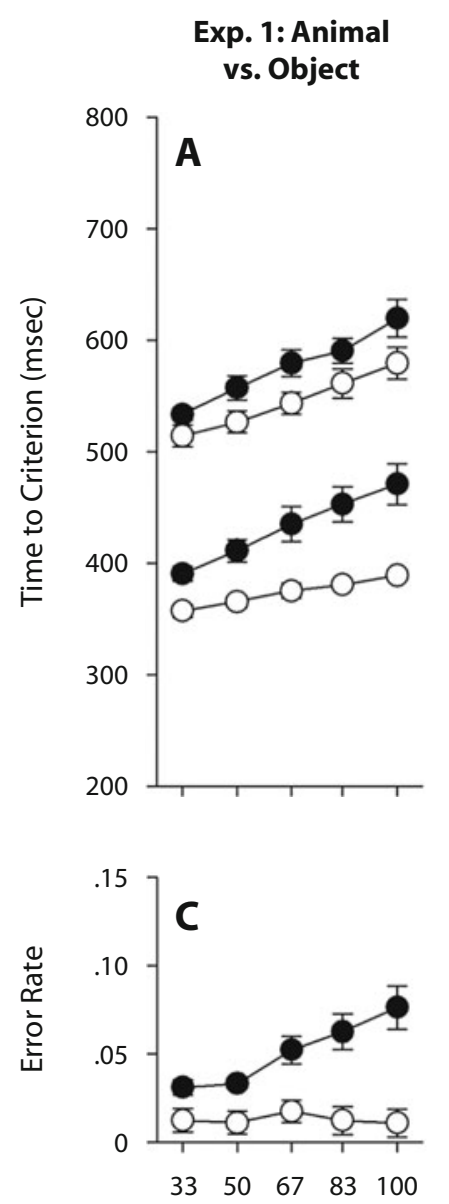

\section{Exp. 1: Large vs. Small}
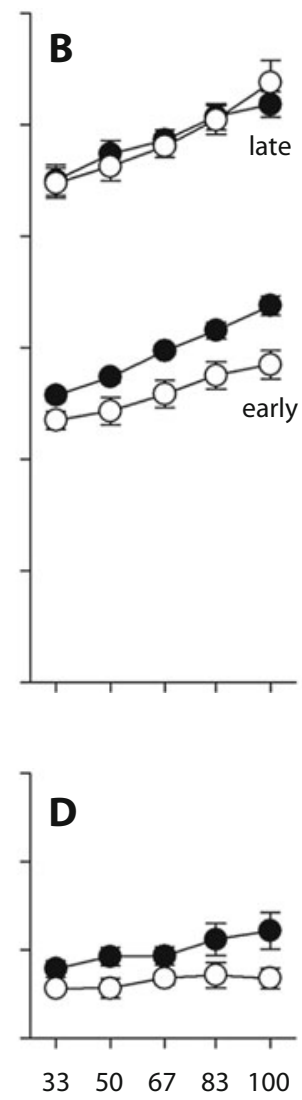

Exp. 2: Toy Animal vs. Object
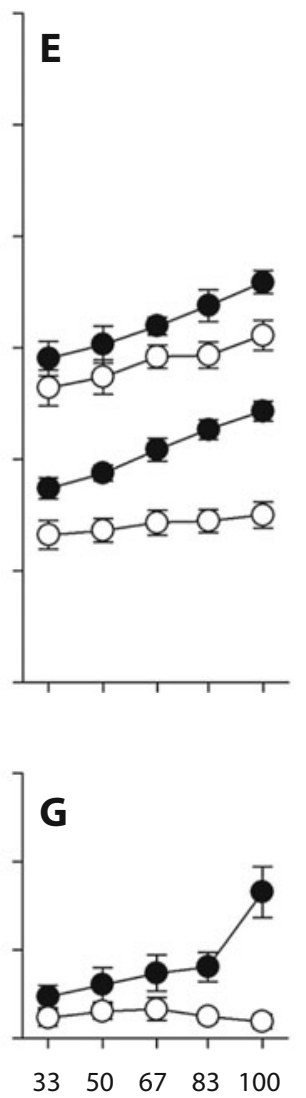

\section{Exp. 2: Ellipse vs. Rectangle}
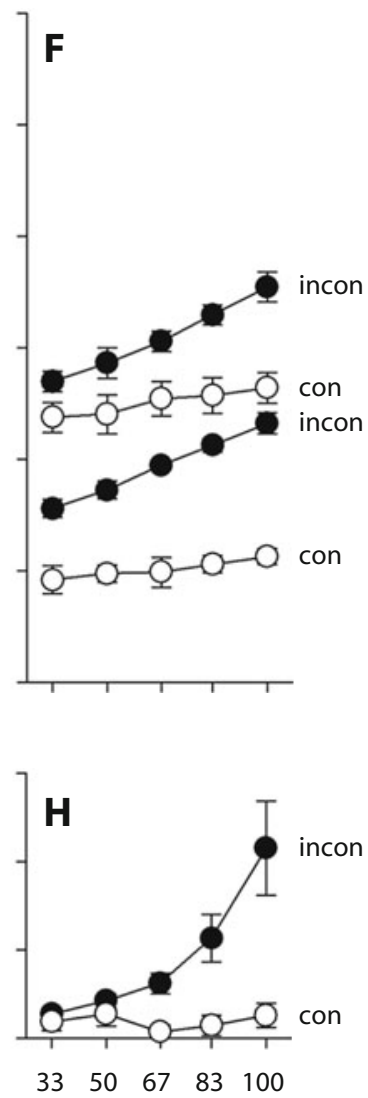

Prime-Target SOA (msec)

Figure 3. Upper panels: Crossing times for the early and late criteria shown in Figure 2 (correct responses only). Lower panels: Rates of response errors, which are defined as arrivals of the sensor at the nontarget position. Error bars are corrected for between-subjects variance (Bakeman \& McArthur, 1996). Incon, inconsistent; con, consistent.

animal-object task (Figure $2 \mathrm{~A}$ ) and roughly $380 \mathrm{msec}$ after prime onset in the large-small task (Figure 2B). The movement was strongly delayed in inconsistent trials, and this delay increased with prime-target SOA. In addition, trajectories were increasingly delayed for longer SOAs in both consistent and inconsistent trials.

Priming effects in the time domain. Temporal priming effects were compared for the consistent and inconsistent trials according to two spatial criteria: when the trajectory had first moved at least $3 \mathrm{~mm}$ in the positive direction (early criterion) and when it first entered a radius of $17 \mathrm{~mm}$ around the correct target position just prior to arrival (late criterion). We employed two separate criteria because earlier studies had shown that the magnitude of temporal priming effects varies over the time course of the response (Schmidt, 2002; Schmidt et al., 2006; Schmidt \& Seydell, 2008). In both the animal-object and large-small tasks, the early criterion was crossed earlier in consistent than in inconsistent trials [at crossing times $t_{\text {con }}$ and $t_{\text {incon }} ; F \mathrm{~s}(1,5)=$ 17.49 and $12.07, p s=.009$ and .018 , respectively]. This priming effect $\left(t_{\text {incon }}-t_{\text {con }}\right)$ increased with prime-target SOA in the animal-object task $[F(4,20)=7.50, p<.001$; see Figure 3A], but not in the large-small task $[F(4,20)=$ $1.08, p=.395$; see Figure $3 \mathrm{~B}]$. In the animal-object task, the late criterion was crossed earlier in consistent than in inconsistent trials $[F(1,5)=31.36, p=.003]$, but this priming effect did not increase with SOA $[F(4,20)=0.76, p=$ .563 ; see Figure 3A]. That late in the trajectory, temporal priming effects in the large-small task had actually disappeared $[F(1,5)<0.01$; see Figure $3 \mathrm{~B}]$. As compared with the early criterion, priming effects were diminished in the large-small task $[F(1,5)=5.67, p=.063]$, but not in the animal-object task $[F(1,5)=2.34, p=.186]$. The analysis of response errors (i.e., arrivals at the wrong target location) corroborated this picture for the animal-object task, for which errors occurred almost exclusively in inconsistent trials, and there increased strongly with SOA (Figure 3C). Accordingly, analysis of the arcsine-transformed error rates confirmed a main effect of consistency $[F(1,5)=16.44$, $p=.010]$, a main effect of SOA $[F(4,20)=4.33, p=$ $.011]$, and an interaction of both factors $[F(4,20)=7.91$, $p=.001$; all Huynh-Feldt corrected]. In the large-small task, only the main effect of consistency was significant $[F(1,5)=10.21, p=.024]$. 
A

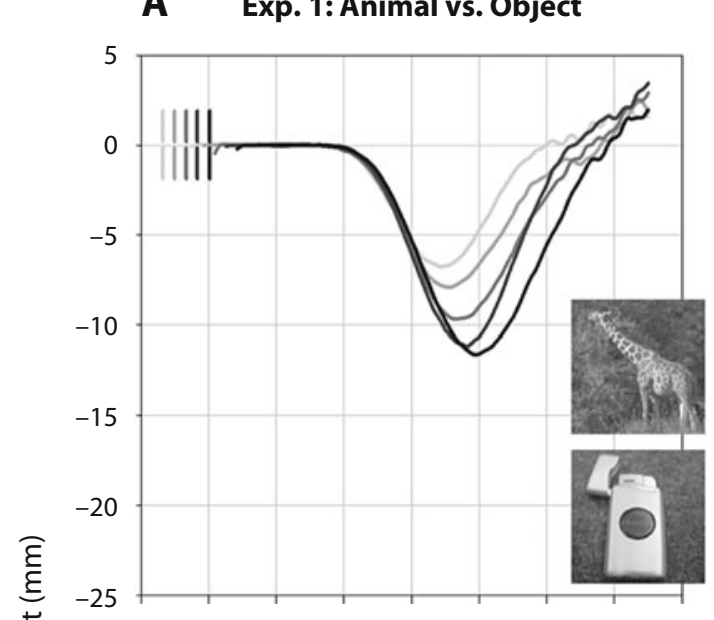

B

Exp. 1: Large vs. Small

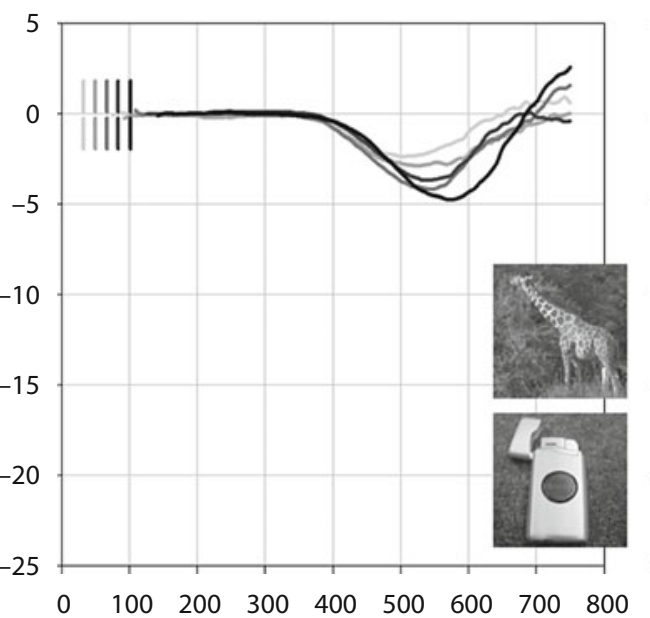

\section{Exp. 2: Toy Animal vs. Object}

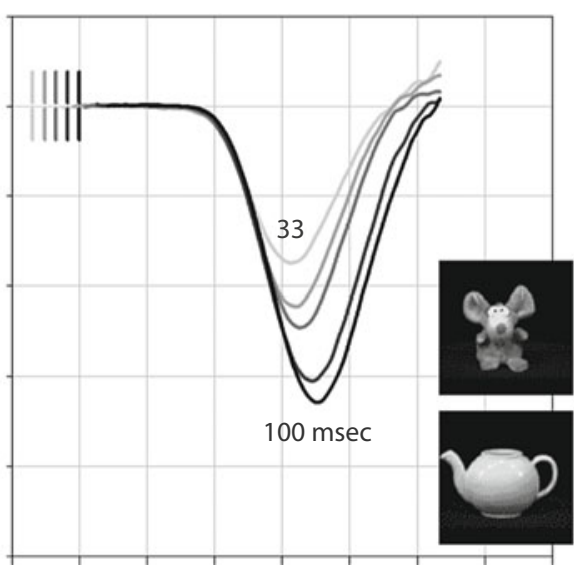

D Exp. 2: Ellipse vs. Rectangle

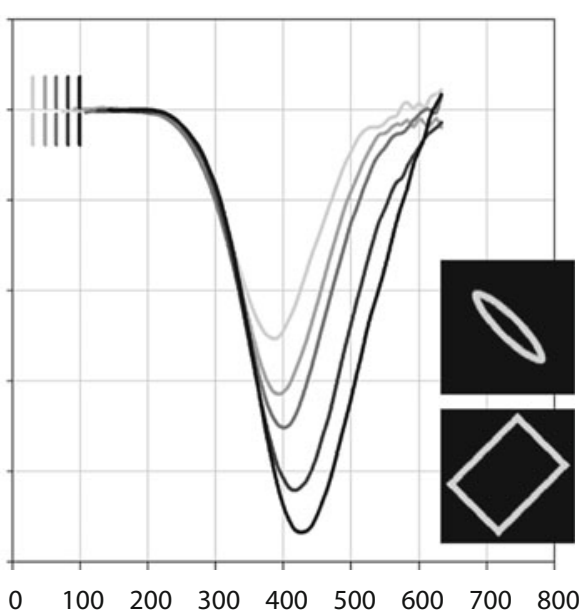

Time From Prime Onset (msec)

Figure 4. Spatial priming functions, derived by subtracting consistent from inconsistent trajectories in Figure 2. Only correct responses are shown. Functions are locked to prime onset. Note that in at least three of the tasks, the initial time course of priming is exactly invariant, as predicted by the rapid-chase model.

Spatial priming functions. In the animal-object task, the time course of spatial priming effects (Figure 4A) conformed to the pattern predicted by the rapid-chase model (Figure 1C). Spatial priming functions were strictly timelocked to prime onset, in line with the idea that the priming effect was actually triggered by that stimulus. Crucially, the early time course of spatial priming was invariant for the different SOAs: All curves initially follow the time course of the curve for the longest SOA, in line with the independence criterion, and branch off only later one by one in the order of increasing SOAs. This pattern appeared less clear in the large-small task, where spatial priming effects were much smaller overall (Figure 4B).

Once the spatial priming functions started to depart from the common time course, they strongly depended on SOA (Figure 5). Onset times of spatial priming effects were defined as those times (from prime onset) when the effect first fell below $-0.5 \mathrm{~mm}$ (meaning that the sensor posi- tion in inconsistent trials lagged at least half a millimeter behind that in consistent trials). This criterion was crossed about $80 \mathrm{msec}$ earlier in the animal-object task than in the large-small task $[F(1,5)=112.19, p<.001]$ but was independent of SOA $[F(4,20)=1.14, p=.369]$. As the primetarget SOA increased, the peak amplitude of the priming function was reached later $[F(4,20)=2.83, p=.052]$ and became more negative $[F(4,20)=5.31, p=.004]$. Overall, the priming function developed more vigorously in the animal-object task than in the large-small task: The peak velocity (i.e., first time derivative) of the spatial priming function was more negative $[F(1,5)=51.75, p<.001]$ and was reached earlier $[F(1,5)=29.79, p=.003]$.

\section{Experiment 2: Toy-Animal-Object and Ellipse-Rectangle Tasks}

The stimuli in Experiment 2 were designed to yield larger priming effects that would develop earlier and more 


\section{Experiment 1: Kinematic Parameters of Spatial Priming Functions}
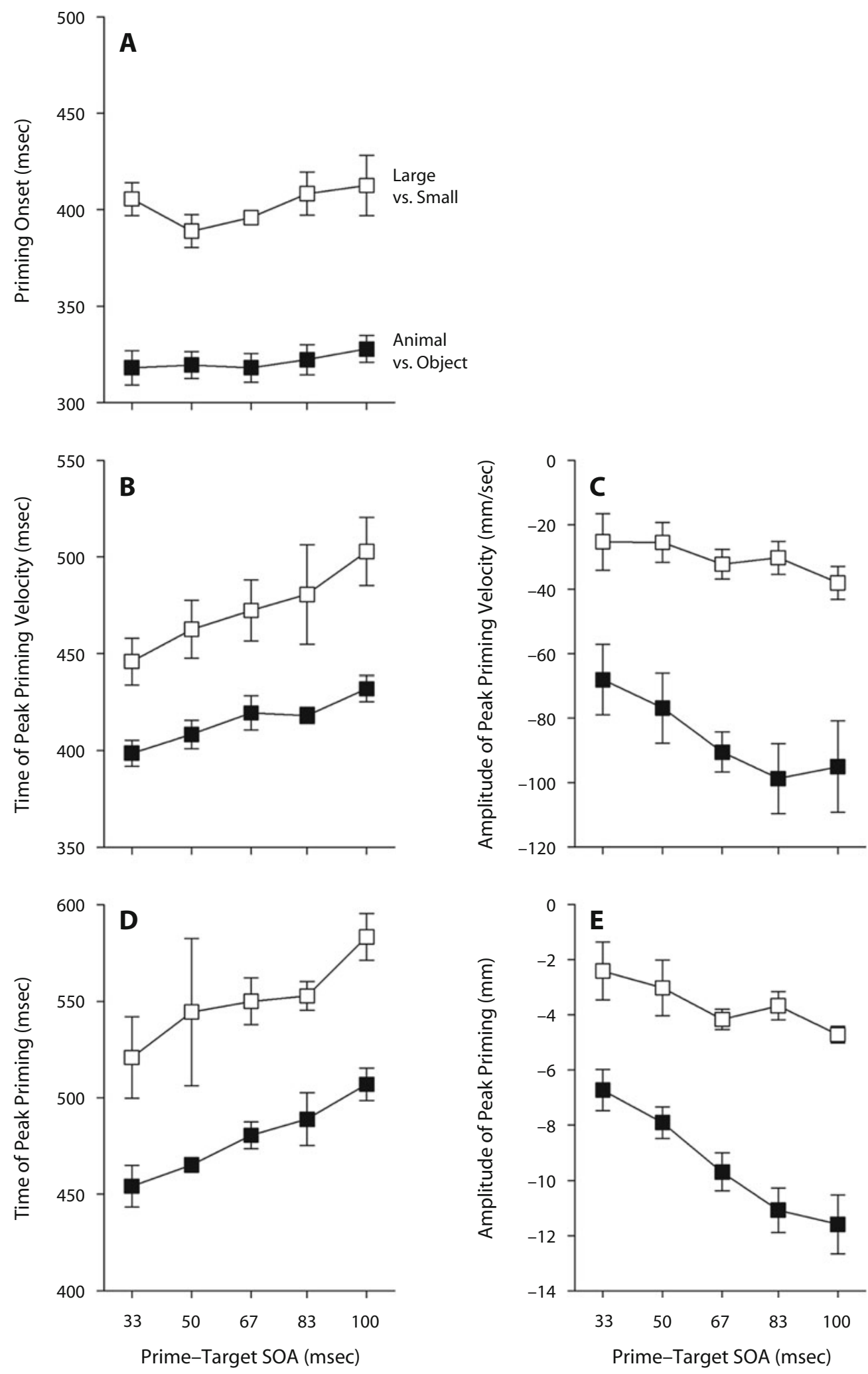

Figure 5. Kinematic parameters of spatial priming functions in the animal-object and large-small tasks in Experiment 1. (A) Onset times of spatial priming effects. (B) Time at which the priming function developed at peak velocity. (C) Amplitude of priming effects at peak velocity. (D) Time of peak priming effects. (E) Amplitude of peak priming effects. 
vigorously than those in Experiment 1. Indeed, movements started earlier, roughly $280 \mathrm{msec}$ after prime onset in the toy-animal-object task and roughly $220 \mathrm{msec}$ after prime onset in the ellipse-rectangle task (Figures 2C and 2D). On average, the initial movement direction clearly followed the primes rather than the targets. In consistent trials, this was the correct direction; the sensor traveled continuously in the direction of the correct target until the response was completed. In inconsistent trials, however, the sensor tended to detour into the quadrant specified by the misleading prime before reversing and proceeding in the correct direction. This detour was longer and reached further into the wrong quadrant when more time had elapsed between the primes and targets. Note that in contrast to the simple delays observed in Experiment 1, actual detours toward the misleading prime are an unambiguous indication that the prime initially controls the response.

Priming effects in the time domain. In both the toyanimal-object and ellipse-rectangle task, the early criterion was crossed earlier in consistent than in inconsistent trials $\left[F_{\mathrm{s}}(1,5)=19.20\right.$ and $74.48, p=.007$ and $p<.001$, respectively; see Figures $3 \mathrm{D}$ and $3 \mathrm{~F}]$. This priming effect increased with prime-target SOA in both tasks $[F \mathrm{~S}(4,20)=$ 5.80 and $11.42, p=.003$ and $p<.001$, respectively]. The late criterion was crossed earlier in consistent than in inconsistent trials in both the toy-animal-object and the ellipse-rectangle tasks $\left[F_{\mathrm{S}}(1,5)=13.23\right.$ and 45.94, $p \mathrm{~s}=.015$ and .001 , respectively; see Figures $3 \mathrm{E}$ and $3 \mathrm{~F}]$. These priming effects increased with prime-target SOA in both tasks $[F \mathrm{~s}(4,20)=2.95$ and $7.41, p=.045$ and $p<.001$, respectively]. Additional analyses confirmed a reduction in priming effects at the late criterion, as compared with the early criterion, for the toy-animal-object task, as well as for the ellipse-rectangle task $[F \mathrm{~s}(1,5)=$ 6.77 and $12.00, p s=.048$ and .018 , respectively]. The analysis of response errors (Figures $4 \mathrm{G}$ and $4 \mathrm{H}$ ) corroborated this picture: In both tasks, errors occurred almost exclusively in inconsistent trials, and there, error rates increased strongly with SOA. Accordingly, in both the toy-animal-object and the ellipse-rectangle tasks, there were significant effects of consistency $\left[F_{\mathrm{S}}(1,5)=10.11\right.$ and $13.45, p \mathrm{~s}=.025$ and .014 , respectively], and of SOA $[F \mathrm{~s}(4,20)=4.35$ and $12.16, p=.011$ and $p<.001$, respectively] and interactions of both factors $\left[F_{\mathrm{s}}(4,20)=\right.$ 7.89 and $6.52, p \mathrm{~s}=.003$ and .007 , respectively].

Spatial priming functions. The time course of spatial priming effects (Figures 4C and 4D) conformed to the pattern predicted by the rapid-chase model, with priming functions strictly time-locked to prime onset and a strict invariance of the early time courses of priming. Again, all the curves initially followed the time course of the curve for the longest SOA. Once the curves started to depart from the common time course, spatial priming effects strongly depended on SOA (Figure 6). Priming onset occurred about $60 \mathrm{msec}$ earlier in the ellipse-rectangle task than in the toy-animal-object task [even though this effect was not significant; $F(1,5)=2.95, p=.147]$, and onset times were independent of SOA $[F(4,20)=1.81$, $p=.166]$. As the prime-target SOA increased, the peak amplitude of the priming function was reached later
$[F(4,20)=9.16]$ and became more negative $[F(4,20)=$ 27.20 ; both $p \mathrm{~s}<.001]$. Overall, the priming function developed more vigorously in the ellipse-rectangle task than in the toy-animal-object task: The peak velocity (i.e., first time derivative) of the spatial priming function was more negative $[F(1,5)=5.42, p=.067]$ and was reached ear$\operatorname{lier}[F(1,5)=8.61, p=.033]$.

\section{DISCUSSION}

Response priming effects for natural images and geometrical shapes are similar to those obtained with simple color stimuli (Schmidt, 2002; Schmidt et al., 2006; Schmidt \& Seydell, 2008). Generally, responses start at a fixed time following prime onset and initially go into the direction specified by the primes, suggesting that the primes initiate the response, instead of the actual targets. When primes and targets are consistent, this initial direction is correct, and the sensor simply travels toward the correct target until the response is completed. When primes and targets are inconsistent, the sensor initially detours into the quadrant of the misleading primes. This detour lasts for a time depending on prime-target SOA; then the movement reverses and finally proceeds in the correct direction, obviously because the response is now controlled by the targets. Even in the absence of overt detours, misleading response initiation by inconsistent primes delays response activation in the correct direction, and these delays increase with prime-target SOA. Importantly, priming effects are also present in the pattern of response errors - that is, prime-triggered responses that actually reach the wrong target location. Errors occur mainly in inconsistent trials, and there increase with SOA, further showing that the impact of the primes goes all the way to the level of motor processing (Vorberg et al., 2003).

These findings are consistent with a feedforward processing system. Indeed, the toy-animal-object and the ellipse-rectangle tasks clearly satisfy the rapid-chase criteria (Schmidt et al., 2006; Vath \& Schmidt, 2007): Initial responses are time-locked to prime onset and go in the direction specified by the primes (initiation criterion); responses are taken over in midflight by the target signals (takeover criterion); and most important, responses initiated by the prime signals are initially independent of the onset times of the targets (independence criterion). As is shown in Figure 4, spatial priming functions in each task are initially invariant when locked to prime onset, perfectly following exactly the same initial time course before individual priming functions branch off one after another in the order of increasing prime-target SOA.

This finding contradicts any model of priming in which the response right at the onset of the priming effect is controlled by information coming from the target as well as the prime, indicating some mixture of signals prior to response onset. Under such a model, initial response activation in the direction of the prime should become weaker for shorter prime-target SOAs, because this factor should increase the influence of the target signal relative to the prime signal and thus reduce the priming effect (as in Figure 1D). In contrast, the finding that this time course is 


\section{Experiment 2: Kinematic Parameters of Spatial Priming Functions}
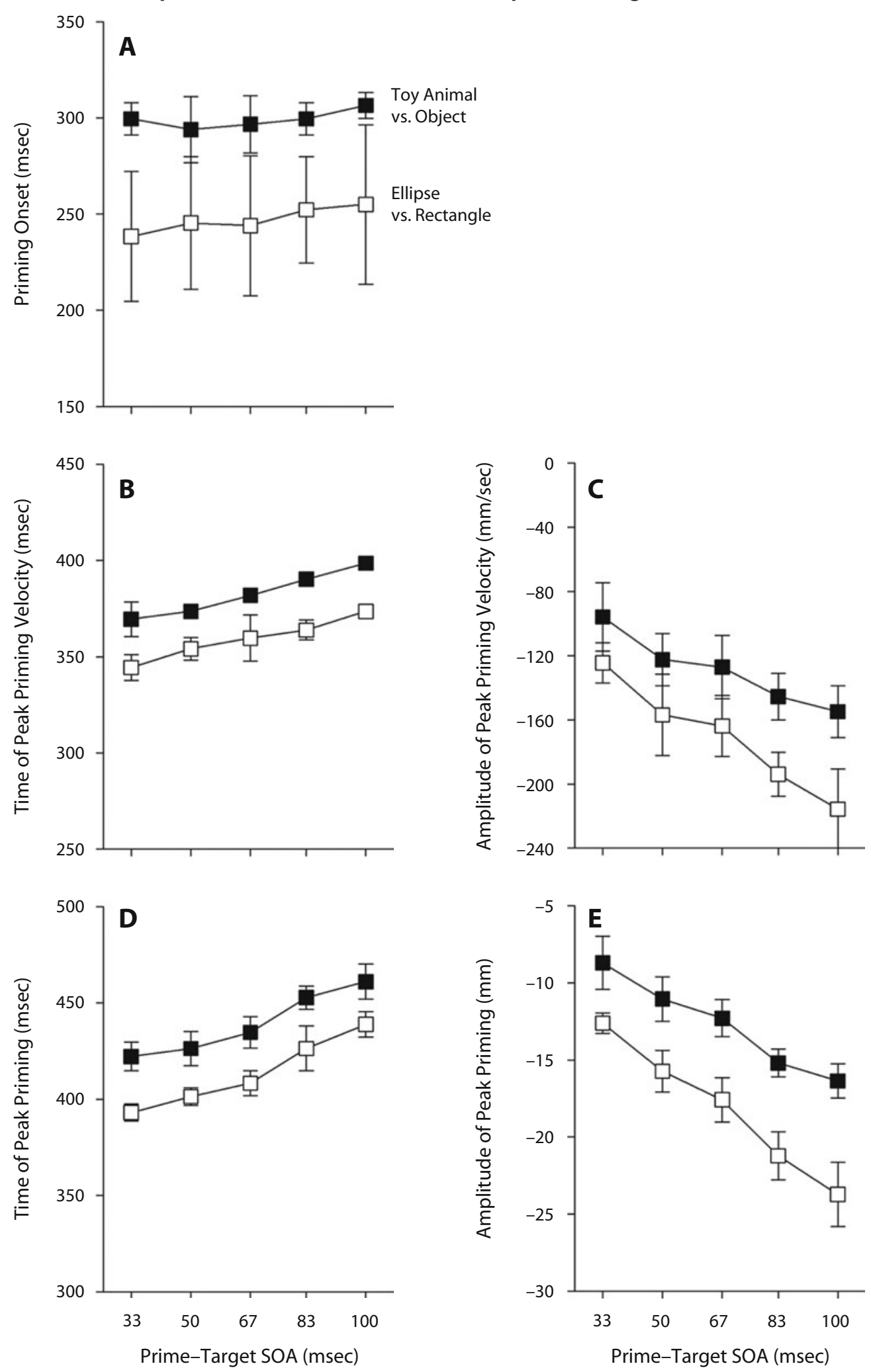

Figure 6. Kinematic parameters of spatial priming functions in the toy-animal-object and ellipserectangle tasks in Experiment 2. (A) Onset times of spatial priming effects. (B) Time at which the priming function developed at peak velocity. (C) Amplitude of priming effects at peak velocity. (D) Time of peak priming effects. (E) Amplitude of peak priming effects. 
initially invariant (as in Figure 1C) indicates that the target signal has no influence whatsoever at the time when the prime first affects the response, which indicates that early priming effects are based on signals carrying only prime but no target information.

Admittedly, these issues are less clear in Experiment 1, in which both tasks failed to meet the initiation criterion, at least when judged by looking only at the raw trajectories (Figure 2). In comparison with the high-contrast color images employed in Experiment 2, the grayscale images failed to produce priming effects vigorous enough to provoke overt detours into the quadrant of the misleading prime, which makes it difficult to determine the exact time courses of response-related processes by looking at the trajectories alone. However, we have previously shown (Schmidt et al., 2006) that overt detours occur only in a minority of responses with very fast onset times, whereas in the majority of trials, priming effects manifest themselves in delayed onset times instead of overt detours. Even in the absence of detours, the delay of pointing onset probably reflects a covert conflict of opposing response tendencies from prime and target signals that must be resolved before movement onset can occur; therefore, an absence of overt detours does not imply that initial response activation is not time-locked to the prime. The best way to see this is to look at the spatial priming functions (Figure 4), because these functions measure the impact of the primes after subtracting out the impact of the targets. In the animal-object task in Experiment 1, these functions clearly meet the independence criterion: Functions are time-locked to the prime and are initially invariant no matter what the time of target onset, just as in the corresponding task in Experiment 2. Despite the ambiguity in the raw trajectories, the spatial priming functions thus allow the conclusion that these data satisfy the rapid-chase criteria as well.

Only in the large-small task, with its tardy response dynamics and small priming effects, does it become difficult to evaluate whether the independence criterion is met. But why is the large-small task so slow? It is unlikely that the difference lies in image processing per se: Earlier studies have shown that participants are equally fast at detecting pictures of transportation devices (e.g., cars, planes) and animals (VanRullen \& Thorpe, 2001), just as animals and human faces can be picked out of distractor images about equally quickly (Rousselet, Macé, \& Fabre-Thorpe, 2003). It rather seems that the large-small classification cannot be performed in a single feedforward sweep and requires more extensive, recurrent processing. It is also possible that this task requires cognitive control to overrule a natural tendency to classify the images on the basis of the feedforward distinction between animals and objects.

Our results extend our rapid-chase theory of response priming to the domain of natural image processing, linking visuomotor priming to feedforward processing of images (Schmidt et al., 2006; Vath \& Schmidt, 2007). Rapid-chase theory assumes that prime and target signals are transmitted sequentially by early feedforward waves of visuomotor processing (Bullier, 2001; Lamme \& Roelfsema, 2000) elicited in turn by primes and targets. Prime and target signals are able to directly initiate the motor responses assigned to them, with no need for conscious mediation ( $d i-$ rect parameter specification; Neumann, 1990; Neumann \& Klotz, 1994). The prime signal reaches executive motor areas first, initiating a response and continuing to drive the response on its own. After a delay that depends on the prime-target SOA, the target signal arrives and takes over response control from the prime signal. Priming effects increase with prime-target SOA because the prime has more time to drive the response on its own when the target is further delayed. Response priming may occur overtly, with noticeable detours of response trajectories in the direction of the prime, or covertly, visible only in the delayed onset of movements after response conflicts are solved. Primes inconsistent with the masks will occasionally provoke response errors, particularly if the prime-mask SOA is long, the prime signal is strong, and the motor response happens to start quickly after prime onset (Schmidt et al., 2006; Vath \& Schmidt, 2007; Vorberg et al., 2003). Transmission of prime signals is assumed to be fast enough to escape recurrent degradation of the prime signal by visual masking (DiLollo et al., 2000; Fahrenfort et al., 2007; Lamme, 2002; Lamme et al., 2002), so that priming effects remain unaffected even when the prime is heavily masked (Bacon-Macé et al., 2005; Schmidt \& Vorberg, 2006; Vorberg et al., 2003).

Our data strongly support the conclusion from previous studies (e.g., Bacon-Macé et al., 2007; Kirchner \& Thorpe, 2006; Thorpe et al., 1996; VanRullen \& Koch, 2003; VanRullen \& Thorpe, 2001, 2002) that natural scenes can be classified on the basis of an initial feedforward sweep of processing (Bullier, 2001; Lamme \& Roelfsema, 2000). However, we make no claim here that classification is based exclusively on the semantic content of the images. For the grayscale images used in Experiment 1, it has been argued that the animal-nonanimal classification is unlikely to be based on differences in low-level image features (e.g., spatial frequency spectra; Kirchner \& Thorpe, 2006). On the other hand, it has been shown that in rapid serial visual presentations of images, participants can be very quick in detecting a target category but still have difficulties identifying and localizing it, implying that the images are not fully processed (Evans \& Treisman, 2005). Whatever information is ultimately used to guide image classification, our data indicate a rapid-chase system (Schmidt et al., 2006; Vath \& Schmidt, 2007) that is behaviorally equivalent to a feedforward system with high temporal resolution. Our data thus suggest that visuomotor priming effects can capture the output of the very first pass of information through the visuomotor system, prior to contributions from recurrent processing.

However, we believe that this conclusion cannot be reached by looking only at the response time distributions of responses to single stimuli (or stimulus pairs); instead, the crucial issue is whether stimulus signals remain separate when traversing the visuomotor system in sequence, thus satisfying stringent predictions of a feedforward model. This is actually an important theoretical point, because it links feedforward processing to functional char- 
acteristics of the processing system, not its overall speed. If we consider the different tasks described here, we can see that they differ markedly in the speed of response onsets and the amplitude of priming effects: The ellipserectangle task produced the fastest responses and largest priming effects, with an onset of spatial priming effects at roughly $220 \mathrm{msec}$ after prime onset, whereas priming effects in the toy-animal-object task in Experiment 2 started about $60 \mathrm{msec}$ later; those in the corresponding task in Experiment 1 were slower still; and those in the largesmall task developed a full $160 \mathrm{msec}$ after those in the ellipse-rectangle task. These differences considered, there is no indication here that natural image classification is extraordinarily fast, or "ultra-rapid" (Kirchner \& Thorpe, 2006; VanRullen \& Thorpe, 2001), when compared with even more basic tasks such as simple shape classification. But even though the animal-object and ellipse-rectangle tasks reported here represent a wide range of processing speeds, they all meet strict functional criteria for the behavior of a rapid-chase system (with some hint at rapidchase processing even for the "ultra-slow" large-small task). It is therefore important to stress that a feedforward system is characterized not only by the sheer rapidity of its processing, but also by its temporal dynamics when sequential stimulus information is processed, even if the rate of processing is comparatively slow.

\section{AUTHOR NOTE}

This research was supported by the German Research Foundation, Grant Schm1671/1 to T.S. The authors declare that they have no competing financial interests. Correspondence concerning this article should be addressed to T. Schmidt, Department of Experimental and Cognitive Psychology, University of Giessen, Otto-Behaghel-Str. 10F, D-35394 Giessen, Germany (e-mail: thomas.schmidt@psychol.uni-giessen.de).

\section{REFERENCES}

Bacon-MacÉ, N., Kirchner, H., FAbre-Thorpe, M., \& Thorpe, S. J. (2007). Effects of task requirements on rapid natural scene processing: From common sensory encoding to distinct decisional mechanisms. Journal of Experimental Psychology: Human Perception \& Performance, 33, 1013-1026. doi:10.1037/0096-1523.33.5.1013

Bacon-Macé, N., Macé, M. J.-M., Fabre-Thorpe, M., \& Thorpe, S. J. (2005). The time course of visual processing: Backward masking and natural scene categorisation. Vision Research, 45, 1459-1469. doi:10.1016/j.visres.2005.01.004

BaKeman, R., \& MCArthur, D. (1996). Picturing repeated measures: Comments on Loftus, Morrison, and others. Behavior Research Methods, Instruments, \& Computers, 28, 584-589.

BULLIER, J. (2001). Integrated model of visual processing. Brain Research Reviews, 36, 96-107. doi:10.1016/S0165-0173\%2801\%2900085-6

Dehaene, S., Naccache, L., Le Clec'H, G., Koechlin, E., MuelLeR, M., Dehaene-Lambertz, G., ET AL. (1998). Imaging unconscious semantic priming. Nature, 395, 597-600. doi:10.1038/26967

DiLollo, V., Enns, J. T., \& Rensink, R. A. (2000). Competition for consciousness among visual events: The psychophysics of reentrant visual processes. Journal of Experimental Psychology: General, 129, 481-507. doi:10.1037/0096-3445.129.4.481

Eimer, M., \& SchlaghecKen, F. (1998). Effects of masked stimuli on motor activation: Behavioral and electrophysiological evidence. Journal of Experimental Psychology: Human Perception \& Performance, 24, 1737-1745. doi:10.1037/0096-1523.24.6.1737

Evans, K. K., \& Treisman, A. (2005). Perception of objects in natural scenes: Is it really attention-free? Journal of Experimental Psychology: Human Perception \& Performance, 31, 1476-1492. doi:10.1037/0096 $-1523.31 .6 .1476$
FAhrenfort, J. J., Scholte, H. S., \& LAmme, V. A. F. (2007). Masking disrupts reentrant processing in human visual cortex. Journal of Cognitive Neuroscience, 19, 1488-1497. doi:10.1162/jocn.2007.19 .9 .1488

Geusebroek, J. M., Burghouts, G. J., \& Smeulders, A. W. M. (2005). The Amsterdam library of object images. International Journal of Computer Vision, 61, 103-112. doi:10.1023/B:VISI.0000042993 .50813 .60

Hegdé, J. (2008). Time course of visual perception: Coarse-to-fine processing and beyond. Progress in Neurobiology, 84, 405-439. doi:10.1016/j.pneurobio.2007.09.001

JAŚKowsKI, P., \& ŚLÓSAREK, M. (2007). How important is a prime's gestalt for subliminal priming? Consciousness \& Cognition, 16, 485497. doi:10.1016/j.concog.2006.06.005

KIRCHNER, H., \& THORPE, S. J. (2006). Ultra-rapid object detection with saccadic eye movements: Visual processing speed revisited. Vision Research, 46, 1762-1776. doi:10.1016/j.visres.2005.10.002

Lamme, V. A. F. (2002). Neural mechanisms of visual awareness: A linking proposition. Brain \& Mind, 1, 385-406. doi:10.1023/ A:1011569019782

Lamme, V. A. F., Rodriguez-Rodriguez, V., \& Spekreisse, H. (1999). Separate processing dynamics for texture elements, boundaries and surfaces in primary visual cortex. Cerebral Cortex, 9, 406-413. doi:10.1093/cercor/9.4.406

Lamme, V. A. F., \& Roelfsema, P. R. (2000). The distinct modes of vision offered by feedforward and recurrent processing. Trends in Neurosciences, 23, 571-579. doi:10.1016/S0166-2236(00)01657-X

Lamme, V. A. F., Zipser, K., \& SpekreiJse, H. (2002). Masking interrupts figure-ground signals in V1. Journal of Cognitive Neuroscience, 14, 1044-1053. doi:10.1162/089892902320474490

Lleras, A., \& ENNS, J. T. (2004). Negative compatibility or object updating? A cautionary tale of mask-dependent priming. Journal of Experimental Psychology: General, 133, 475-493. doi:10.1037/0096 $-3445.133 .4 .475$

Merigan, W. H., \& Maunsell, J. H. R. (1993). How parallel are the primate visual pathways? Annual Review of Neuroscience, 16, 369402. doi:10.1146/annurev.ne.16.030193.002101

Neumann, O. (1990). Direct parameter specification and the concept of perception. Psychological Research, 52, 207-215. doi:10.1007/ BF00877529

Neumann, O., \& KLotz, W. (1994). Motor responses to nonreportable, masked stimuli: Where is the limit of direct parameter specification? In C. Umiltà \& M. Moscovitch (Eds.), Attention and performance XV: Conscious and nonconscious information processing (pp. 124-150). Cambridge, MA: MIT Press.

Pascual-Leone, A., \& Walsh, V. (2001). Fast backprojections from the motion to the primary visual area necessary for visual awareness. Science, 292, 510-512. doi:10.1126/science. 1057099

Ro, T., Breitmeyer, B., Burton, P., Singhal, N. S., \& Lane, D. (2003). Feedback contributions to visual awareness in human occipital cortex. Current Biology, 11, 1038-1041. doi:10.1016/S0960 $-9822(03) 00337-3$

Roelfsema, P. R., Tolboom, M., \& Khayat, P. S. (2007). Different processing phases for features, figures, and selective attention in primary visual cortex. Neuron, 56, 785-792. doi:10.1016/j .neuron.2007.10.006

Rousselet, G. A., Macé, M. J.-M., \& Fabre-Thorpe, M. (2003). Is it an animal? Is it a human face? Fast processing in upright and inverted natural scenes. Journal of Vision, 3, 440-455. doi:10.1167/3.6.5

SchmidT, T. (2002). The finger in flight: Real-time motor control by visually masked color stimuli. Psychological Science, 13, 112-118. doi:10.1111/1467-9280.00421

Schmidt, T., Niehaus, S., \& Nagel, A. (2006). Primes and targets in rapid chases: Tracing sequential waves of motor activation. Behavioral Neuroscience, 120, 1005-1016. doi:10.1037/0735 $-7044.120 .5 .1005$

SCHMidT, T., \& SeYdell, A. (2008). Visual attention amplifies response priming of pointing movements to color targets. Perception \& Psychophysics, 70, 443-455. doi:10.3758/PP.70.3.443

Schmidt, T., \& Vorberg, D. (2006). Criteria for unconscious cognition: Three types of dissociation. Perception \& Psychophysics, 68, 489-504.

Serre, T., Oliva, A., \& Poggio, T. (2007). A feedforward architecture 
accounts for rapid categorization. Proceedings of the National Academy of Sciences, 104, 6424-6429. doi:10.1073/pnas.0700622104

Thorpe, S. J., Fize, D., \& MARLOT, C. (1996). Speed of processing in the human visual system. Nature, 381, 520-522. doi:10.1038/381520a0

Tong, F. (2003). Primary visual cortex and visual awareness. Nature Reviews Neuroscience, 4, 219-229. doi:10.1038/nrn1055

UlRich, R., \& MilleR, J. (2001). Using the jackknife-based scoring method for measuring LRP onset effects in factorial designs. Psychophysiology, 38, 816-827. doi:10.1111/1469-8986.3850816

VanRullen, R., Delorme, A., \& Thorpe, S. J. (2001). Feed-forward contour integration in primary visual cortex based on asynchronous spike propagation. Neurocomputing, 38-40, 1003-1009.

VanRullen, R., Gautrais, J., Delorme, A., \& Thorpe, S. J. (1998). Face processing using one spike per neuron. Biological Systems, 48, 229-239. doi:10.1016/S0303-2647(98)00070-7

VAnRullen, R., \& KoCH, C. (2003). Visual selective behaviour can be triggered by a feed-forward process. Journal of Cognitive Neuroscience, 15, 209-217. doi:10.1162/089892903321208141

VANRullen, R., \& Thorpe, S. J. (2001). Is it a bird? Is it a plane? Ultra-rapid visual categorisation of natural and artifactual objects. Perception, 30, 655-668. doi:10.1068/p3029

VANRullen, R., \& THORPE, S. J. (2002). Surfing a spike wave down the ventral stream. Vision Research, 42, 2593-2615. doi:10.1016/S0042 $-6989(02) 00298-5$

VAth, N., \& Schmidt, T. (2007). Tracing sequential waves of rapid visuomotor activation in lateralized readiness potentials. Neuroscience, 145, 197-208. doi:10.1016/j.neuroscience.2006.11.044

Velleman, P. F. (1980). Definition and comparison of robust nonlinear data smoothing algorithms. Journal of the American Statistical Association, 75, 609-615.

Verleger, R., Jaśkowski, P., Aydemir, A., van der Lubbe, R., \& Groen, M. (2004). Qualitative differences between conscious and nonconscious processing? On inverse priming induced by masked arrows. Journal of Experimental Psychology: General, 133, 494-515. doi:10.1037/0096-3445.133.4.494

Vorberg, D., Mattler, U., Heinecke, A., Schmidt, T., \& Schwarz-
BACH, J. (2003). Different time courses for visual perception and action priming. Proceedings of the National Academy of Sciences, 100, 6275-6280. doi:10.1073/pnas.0931489100

\section{NOTES}

1. To get a feel for how sequential signals control behavior in a rapidchase system, consider the following analogy, modified from a reviewer's suggestion. Imagine that you are the driver of a California stagecoach. Your boss wants you to make a drive to Mexico as quickly as possible, and to instruct you, he sends a messenger on horseback who needs some time to reach you. After your boss has dispatched the messenger, he reconsiders and sends you a second messenger with the instruction to go to Arizona instead. What will you do? If the second messenger has not caught up with the first one, you will leave for Mexico and travel in the wrong direction (initiation criterion), on the basis of the instructions of only the first messenger (independence criterion), until the second messenger reaches you and corrects your course (takeover criterion). Your detour to Mexico will start long after both messages have been dispatched and will last for a time depending on the delay of the second messenger relative to the first one. This system is feedforward in the sense that the messengers are unable to reconcile their messages before exerting control on your behavior.

2. Because we were interested in the motor effects of primes and targets, we decided against reducing the visibility of the primes by additional masking stimuli, because masks inserted between primes and targets seem to impact responses at the motor level (Jaśkowski \& Ślósarek, 2007; Lleras \& Enns, 2004; Verleger et al., 2004). More important, our rapid-chase criteria make no predictions for such a three-stimulus sequence. Even though the target images led to substantial backward masking of prime images, especially at short SOAs, no claim is made here for unconscious processing of the primes.

(Manuscript received July 18, 2008; revision accepted for publication October 12, 2008.) 